\title{
PRODUCING A LINEAR LASER SYSTEM FOR 3D MODELIMG OF SMALL OBJECTS
}

\author{
A. Sh. Amini ${ }^{a}$, M. H. Mozaffar ${ }^{b}$ \\ ${ }^{a}$ Department of Geomatic, South Tehran Branch, Islamic Azad University, Tehran, Iran, sh_amini@azad.ac.ir \\ ${ }^{\mathrm{b}}$ Faculty of Geodesy and Geomatics, K.N. Toosi University of Technology, Tehran, Iran, \\ morteza_heidarymozaffar@yahoo.com
}

Commission V, WG V/5

KEYWORDS: 3D Modeling, Calibration, Laser line, Triangulation

\begin{abstract}
Today, three dimensional modeling of objects is considered in many applications such as documentation of ancient heritage, quality control, reverse engineering and animation In this regard, there are a variety of methods for producing three-dimensional models. In this paper, a 3D modeling system is developed based on photogrammetry method using image processing and laser line extraction from images. In this method the laser beam profile is radiated on the body of the object and with video image acquisition, and extraction of the laser line from the frames, three-dimensional coordinates of the objects can be achieved. In this regard, first the design and implementation of hardware, including cameras and laser systems was conducted. Afterwards, the system was calibrated. Finally, the software of the system was implemented for three-dimensional data extraction. The system was investigated for modeling a number of objects. The results showed that the system can provide benefits such as low cost, appropriate speed and acceptable accuracy in 3D modeling of objects.
\end{abstract}

\section{INTRODUCTION}

Today, three dimensional modeling of objects is considered in many applications such as documentation of ancient heritage, quality control, reverse engineering and animation [1]. In this regard, there are a variety of methods for producing three-dimensional models. One of the methods of modeling three-dimensional objects is applying laser. The laser is an abbreviation for words "Light Amplification by Simulated Emission of Radiation". A laser device can emits parallel light very narrow, with a specific wavelength. What the laser rises in the issue of three-dimensional modeling of objects is this property. In other words, the laser can be used in many applications as an indicator of an activity, such as navigation and tracking. In 3D modeling applications, a laser scanner, is a device which provides required 3D data for three-dimensional digitization of objects fully or partially [2]. Three-dimensional scanners have numerous applications; consequently they are taking in to consideration by researchers. For example, Canada's National Research Group, developed a sensor named BIRIS and used in many studies [3], [4]. Practical examples of these applications can be found in projects such as documentary journalism of Michelangelo statue [5].

One of the most unsuccessful results in modeling procedure using stereo images is absence or weakness of the tissue on the surface of the object for matching. The main idea of using laser technology in order to achieve three-dimensional information about objects has been established on this basis that instead of processing the data using two camera, a camera and a projector is used to obtain three-dimensional coordinates of object points. These systems named structured light. Figure (1), shows the components of a structured light system.

One of the most effective parameters affects the quality of $3 \mathrm{D}$ model using laser scanners is object's properties. The most influential factors in 3D modeling applying this approach can be mentioned as the color of the object, the material of the object surface, dimension of the object, lightening conditions and static or dynamic modeling method. These parameters must be considered in 3D modeling procedure.

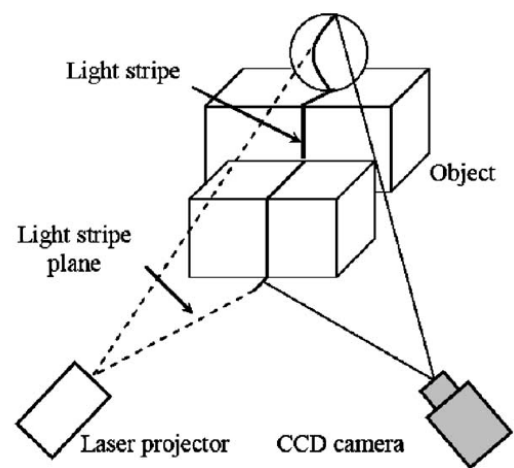

Figure 1. Components of a structured light system

According to the importance of three-dimensional modeling, the goal of this research is design and implementation of a three-dimensional modeling system based on photogrammetry method integration with a linear laser system. In this methodwith radiation of a laser beam on the object, image acquisition and finally extracting laser lines from the images, three-dimensional coordinates of object points can be achieved. Finally, 3D modeling of the object can be presented putting together all of the extracted lines.

In this research, first designing and implementing of a 3D modeling system including a laser device and a camera is introduced. Afterwards, system calibration is explained. In the next stage, details of the laser triangulation method its mathematics, and producing software based on it are described. Finally some objects are modeled applying the system and the results are discussed. 


\section{COMPONENTS OF THE SYSTEM}

A structured light system consists of two main pats: hardware and software. The hardware segment includes implementation and calibration of the system. Producing software segment includes pattern extraction, image processing, and ultimately computation.

\subsection{Hardware}

The hardware of a structured light system consists of a linear laser beam for radiation of a laser line, and a digital camera for image acquisition. In our proposed system, a web cam with model "Logitech HD Webcam C270" is applied. To create a pattern on the surface of the object, a linear laser projector was used. Figure (2) shows the camera and the laser applied in this study.
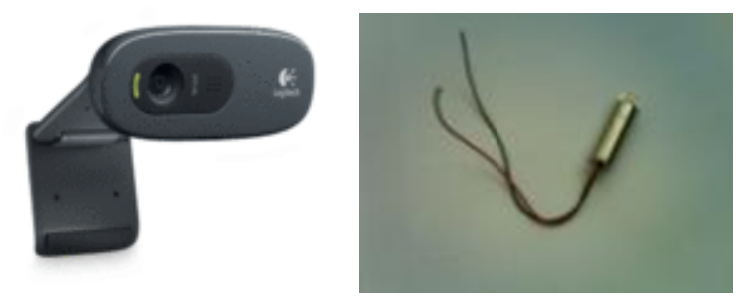

Figure 2. The camera (left) and the laser device (right)

In order to processing the images taken by digital camera and geometric information extraction from, accurate internal camera parameters and also orientation between the laser instrument and the camera is needed. The mentioned camera is a non-metric one; consequently, its accurate calibration is very important. Many methods are presented for camera calibration. In this research, camera calibration is implemented using a test-field. This calibration method is operated based on the use of appropriate set of target coordinates in the object space. Using test-field calibration based on bundle adjustment specifies interior and exterior orientation parameters of the camera plus location and orientation of the laser device relative to the camera. Figure (3) shows test-field used in this study and camera stations in calibration procedure.

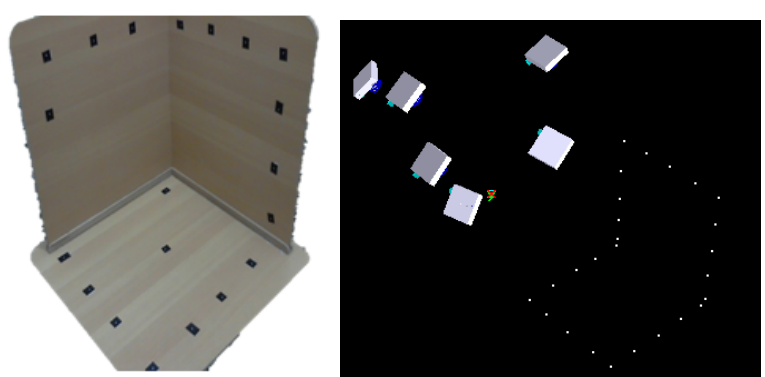

Figure 3. Test-field (left) and camera orientation (right)

After image acquisition from test-field, interior parameters of the camera are computed using Australis software. These parameters are shown in table (1).

\begin{tabular}{|l|l|}
\hline Parameter & Value \\
\hline C $(\mathrm{mm})$ & 6.6673 \\
Xp $(\mathrm{mm})$ & 3.0125 \\
Yp $(\mathrm{mm})$ & 2.1804 \\
$\mathrm{~K} 1$ & $-6.954 \mathrm{e}-004$ \\
$\mathrm{~K} 2$ & $5.065 \mathrm{e}-005$ \\
$\mathrm{~K} 3$ & $0.000 \mathrm{e}+000$ \\
P1 & $6.137 \mathrm{e}-005$ \\
P2 & $-9.853 \mathrm{e}-005$ \\
\hline
\end{tabular}

Table 1. Interior parameters of camera "Logitech HD Webcam C270"

\subsection{Software}

The software of the system consists of implementing two main steps:

1.Recognition of pixels on an image that influenced by the laser line. This issue can be achieved from the difference of two images before and after radiation of laser beam.

2.Using triangulation equations for recognized pixels from previous stage, 3D modeling of the object can be stored or displayed.

Necessary elements for achieving 3D coordinates of an object point from laser beam are shown in figure (4).

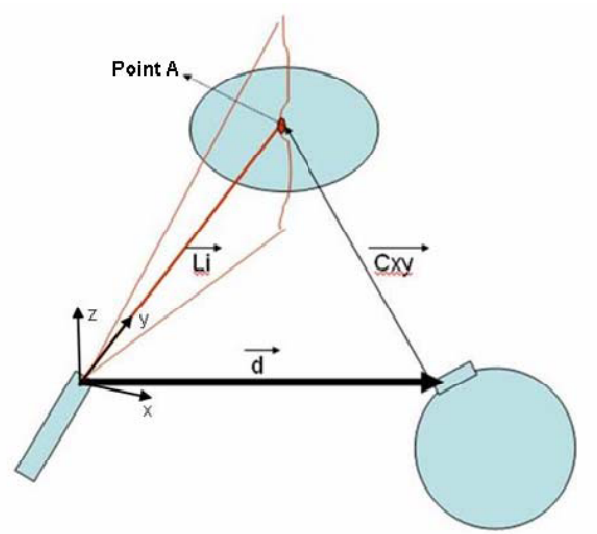

Figure 4. Necessary elements in laser triangulation method

In figure (4), $L_{i}$ is the vector between the origin point and location of striking the laser line in point $A, d$ is the vector between laser line and camera, and $C_{x y}$ is the vector between camera and point $A$. consequently, it can be written as:

$$
\vec{L}_{i}=\vec{d}+\vec{C}_{x y}
$$

In a spherical coordinate system, if we consider $\varphi$ is the angel between laser beam plate and the plate including $Y-Z$ axes, and $\theta$ is the angel between vector $L_{i}$ and axis $Y$, equation (1) can be written as:

$$
\begin{aligned}
& \left|L_{i}\right| \sin \varphi=d_{x}+C_{x y_{x}} \\
& \left|L_{i}\right| \cos \varphi \cos \theta=d_{y}+C_{x y_{y}} \\
& \left|L_{i}\right| \cos \varphi \sin \theta=d_{z}+C_{x y_{z}}
\end{aligned}
$$


For simplicity, if we consider $\varphi=0$, equation (2) can be written as:

$$
\begin{aligned}
& 0=d_{x}+C_{x y_{x}} \\
& L_{y}=\left|L_{i}\right| \cos \theta=d_{y}+C_{x y_{y}} \\
& L_{z}=\left|L_{i}\right| \sin \theta=d_{z}+C_{x y_{z}}
\end{aligned}
$$

And then:

$$
\begin{aligned}
& \left|C_{x y}\right|=\frac{-d_{x}}{\hat{x}} \\
& L_{y}=d_{y}+\left|C_{x y}\right| \hat{y} \\
& L_{z}=d_{z}+\left|C_{x y}\right| \hat{z}
\end{aligned}
$$

In equation (4), $\hat{x}, \hat{y}, \hat{z}$ can be computed from cosine of vector $C_{x y}$. This vector can be obtained from parameters of the camera. After camera calibration and computing interior orientation parameters, it can be written as:

$$
\vec{C}_{x y}=(p x-c x) \hat{i}+(p y-c y) j+f \hat{k}
$$

In equation (5), $(p x, p y)$ is the coordinates of image point, $(c x, c y)$ is the principle point coordinates and $f$ is the focal length. With achieving $C_{x y}$, it can be written:

$$
\hat{x}_{c}=\frac{(p x-c x)}{\left|\hat{C}_{x y}\right|} \quad, \quad \hat{y}_{c}=\frac{(p y-c y)}{\left|\hat{C}_{x y}\right|} \quad, \quad \hat{z}_{c}=\frac{f}{\left|\hat{C}_{x y}\right|}
$$

For exterior orientation parameters, if we present rotation matrix as below:

$$
\left[\begin{array}{l}
\hat{x} \\
\hat{y} \\
\hat{z}
\end{array}\right]=\left[\begin{array}{ccc}
\cos \alpha & 0 & \sin \alpha \\
-\sin \alpha & 0 & \cos \alpha \\
0 & -1 & 0
\end{array}\right]\left[\begin{array}{l}
\hat{x}_{c} \\
\hat{y}_{c} \\
\hat{z}_{c}
\end{array}\right]
$$

Equation (4) can be written as:

$$
\begin{aligned}
& \left|C_{x y}\right|=\frac{-d_{x}}{\hat{z}_{c} \cdot \sin (\alpha)+\hat{x}_{c} \cdot \cos (\alpha)} \\
& L_{y}=d_{y}+\left|C_{x y}\right|\left[-\hat{x}_{c} \cdot \sin (\alpha)+\hat{z}_{c} \cdot \cos (\alpha)\right] \\
& L_{z}=d_{z}+\left|C_{x y}\right|\left(-\hat{y}_{c}\right)
\end{aligned}
$$

In the presented method, the goal is presenting an object coordinate system independent from rotation of the laser device [6]. If laser coordinate system is considered as OUVW and object coordinate system is considered as OXYZ, it can be written:

$$
\left[\begin{array}{c}
r_{x} \\
r_{y} \\
r_{z}
\end{array}\right]=T\left[\begin{array}{c}
r_{u} \\
r_{v} \\
r_{w} \\
1
\end{array}\right]=\left[\begin{array}{c}
0 \\
L_{y} \\
L_{z} \\
1
\end{array}\right]
$$

In this equation, $\left(r_{u}, r_{v}, r_{w}\right)$ is the corresponding triangulation point of $\left(0, L_{y}, L_{z}\right)$. With severing of the transformation equation into two parts including rotation and shift, $T$ can be calculated as:

$$
T=\left[\begin{array}{cccc}
\cos \beta & -\sin \beta & 0 & 0 \\
\sin \beta & \cos \beta & 0 & T_{y} \\
0 & 0 & 1 & T_{z} \\
0 & 0 & 0 & 1
\end{array}\right]
$$

In this equation, $\beta$ is the rotation angle of laser coordinate system.

According to the above contents a computer program is developed in Matlab. In this program, Morphology operators like Erosion and Dilation and also Thickening algorithms are applied in order to thinness of the laser line and finding medial pixels of the laser line. Moreover, Hough Transform operators are also used in order to help finding laser lines on images. In general, stages of 3D modeling with this system can be said as:

1. Image acquisition from test-field in different angles

2. Fixing the camera position in the last station

3. Calculating geometric parameters of the camera

4. Radiating the laser line on the object surface put on the test field, and image capturing with camera

5. Computing the equation of the test-field plate according to the target coordinates

6. Extracting pixels of the laser line on the object surface

7. Triangulation and calculating points coordinates

Figure (5) shows a scheme of the proposed system in 3D modeling of an object. 


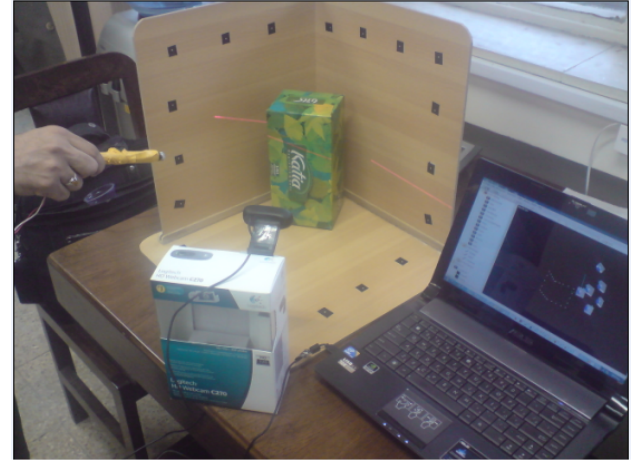

Figure 5. The 3D modeling system

\section{EXPERIMENTS}

After hardware and software implementation of the 3D modeling system, the function of the system is investigated with 3D modeling of some objects. As an instant, a cylindrical object is shown in figure (6).

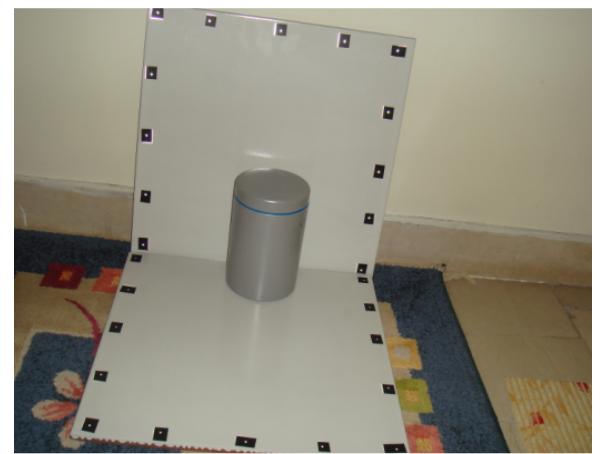

Figure 6. A cylindrical object used for 3D modeling

For cylindrical object, 3D modeling was done in a dark area in order to better recognition and extraction of laser line in images. In each line of the laser, 30 points was extracted. It is possible to increase the number of extracted points with the change of the threshold, but this causes increasing the cost and the time of calculation procedure. Finally, 3D modeling of the cylindrical was performed with about 22000 points. Figure (7) shows the result of $3 \mathrm{D}$ modeling.
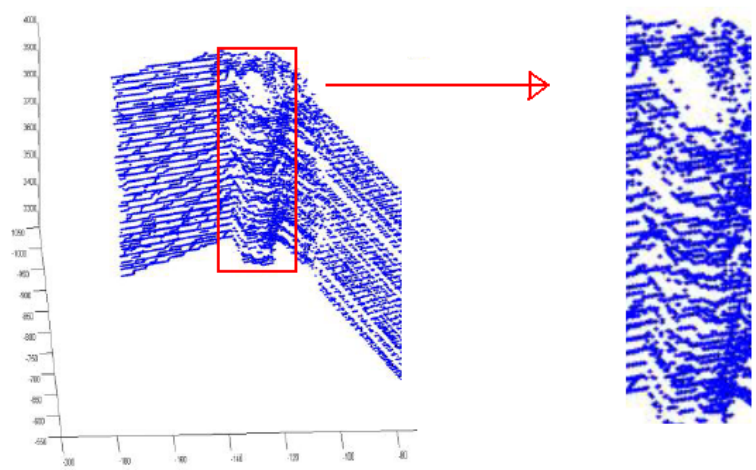

Figure 7. The result of 3D modeling of the cylinder

As it is shown in figure (7), produced 3D modeling of the object is relatively similar to the object shape. In other experiments, similar results were achieved.

With investigating the deficiency of the 3D modeling results, it is concluded that the quality of $3 \mathrm{D}$ models was mostly affected by the quality of the applied hardware including the geometric stability and the resolution of the camera and also the thickness of the laser line and the quality of the results are less affected by the software.

\section{CONCLUSIONS}

The goal of this research is design and implementation of a three-dimensional modeling system based on photogrammetry method integration with a linear laser system. In this method, with radiation of a laser beam on the object, image acquisition and finally extracting laser lines from the images, threedimensional coordinates of object points can be calculated and 3D modeling of the object can be achieved. In general, the mail results of this study are the following:

- The quality of 3D model is mostly affected by the quality of applied hardware including stability and resolution of the camera and the laser device.

- The thickness of the laser line is as the parameters that strongly affects the final result. Consequently the use of lasers lines less thick, better results can be obtained.

- From the other factors affecting the quality of output model, is the properties of the object surface. The material of objects shows different reactions with against the laser. If the material used with high transparency, selecting pixels that are located right on the laser line is difficult due to dispersion.

- It should be mentioned that it is necessary to have uniformity in the laser radiation in order to have time observance between different frames. Since the action of laser radiation was done manually in this study, it created problems on finding points and their continuity. It is proposed to use a device that can automate and regulate the operation of the laser radiation.

- Since laser line is affected by the material of the testfield, it must be selected from materials that have less scattering against laser radiation.

- The angle between laser radiation and camera axis is affects the accuracy of points calculations. Therefore it is recommended this angle is selected less than 30 .

- Using coded targets in calibration procedure can do much to help in automation of the system.

Finally, it can be concluded using appropriate hardware in the system can provide benefits such as low cost, appropriate speed and good accuracy in 3D modeling of objects.

\section{REFERENCES}

[1] Bradshaw G., 1999. Non-Contact Surface Geometry Measurement Techniques. Trinity College Dublin, Department of Computer Science, pp26.

[2] Acosta D., García O., Aponte J., 2006. Laser Triangulation for Shape Acquisition in a $3 D$ Scanner Plus Scanner. Proceedings of the Electronics, Robotics and Automotive Mechanics Conference.

[3] Elgazzar, S., Liscano, R., Blais, F., and Miles, A., 1998. Active Range Sensing for Indoor Environment Modeling. 
IEEE Transactions On Instrumentation And Measurement, Vol. 47, No. 1.

[4] Prieto, F., Redarce, T., Lepage R. y Boulanger, P., 1998 Visual System for Fast and Automated Inspection of $3 d$ Parts. Revue International Conference of Information Graphics, Hermes Science Publications, Paris, Vol. 13, No. 6.

[5] Levoy M., Pulli K., Curless B., Rusinkiewicz S., Koller D., Pereira L., Ginzton M., Anderson S., Davis J.,Ginsberg J., Shade J., Fulk D., 2000. The Digital Michelangelo Project: $3 D$ Scanning of Large Statues. Proceedings of ACM SIGGRAPH, Computer Graphics Proceedings.
[6] Zhou F. , Zhang G., Jie J., 2005. Constructing Feature Points for Calibrating a Structured Light Vision Sensor by Viewing a Lane from Unknown Orientations,.Optics and Lasers in Engineering, Vol 43 , Issue 10, pp 1056-1070.

\section{Acknowledgements}

This investigation is performed as a research project with the title "Producing a Sample of a Linear Laser System for 3D Modeling of Small Objects" under the support of Islamic Azad university, South Tehran branch. 